\title{
The Use of Computational Fluid Dynamics to Study Furnace Effects in ITS-90 Fixed Points Realizations
}

\author{
P. Castro ${ }^{\mathrm{a}^{*}}$, D. del Campo ${ }^{\mathrm{b}}$, R. Lecuna ${ }^{\mathrm{a}}$, C. García Izquierdo ${ }^{\mathrm{b}}$ \\ ${ }^{a}$ Electrical and Energy engineering Department, University of Cantabria, \\ Avda de los Castros, s/n, 39005, Santander, Spain \\ *Corresponding author e-mail: castropb@unican.es \\ ${ }^{b}$ Centro Español de Metrología, Alfar $2 s$ \\ C/Alfar 2, 28760, Tres Cantos, Madrid, Spain \\ e-mail:ddelcampo@cem.minetur.es
}

\begin{abstract}
Together with the impurities, the thermal fluxes are one of the major sources of uncertainty during the realization of the International Temperature Scale of 1990 (ITS-90) defining fixed points. The use of computational fluid dynamics (CFD) is a valuable tool to develop models that describe the time evolution of the phase transformation (essentially the evolution of the solid-liquid interface) as a function of given theoretical assumptions and given parameters (furnace thermal gradients, freezing initiation, ambient temperature and insulation). The models can be validated by observing the impact of the selected parameters on the observed corresponding melting curves and used to achieve a full understanding of these thermal effects and their impact on uncertainty. This paper proposes an ITS-90 metallic fixed points CFD model together with some results about the influence of the furnace thermal gradients and the freezing initiation techniques.
\end{abstract}

Keywords: Computational Fluid Dynamics (CFD); Finite Volume Method (FVM) Software; International Temperature Scale (ITS-90); Fixed Points; Thermal Fluxes; Uncertainty.

\section{Introduction}

Ideally, the best way to realize the fixed point transitions to materialize the ITS-90 is in adiabatic conditions. This is possible with the cryogenic fixed points, but the metallic fixed points, at present, have to be realized using the constant flux technique. This means that they are operated in air using furnaces that usually are at temperatures well below the freezing point temperature (or above in the case of melting points). Therefore it is impossible to reduce the heat fluxes below a few watts.

To minimize this problem, the metal fixed points are often realized with two solid-liquid interfaces (mantles); one is formed on the inner surface of the outside wall of the crucible and an inner interface is formed around the thermometer well. The first one is usually formed by lowering the furnace temperature or by withdrawing the cell from the furnace. The interface around the thermometer well is formed by the insertion of cool rods inside it. The outer interface establishes a temperature controlled zone around the inner interface allowing, in principle, thermal conditions as close as possible to an adiabatic state. But this solution brings with it some complications like the initiation of the freeze (or melting), immersion effects of the standard platinum resistance thermometers and impurity segregation. Some recent works have investigated quasi-adiabatic procedures [1] or new ways to perform the initiation of the plateaus [2], but there are no systematic and complete approaches to shed light on the problem of the thermal effects in the fixed points.

In order to study these thermal effects a two-dimensional (2D) axisymmetric model of the furnace and the fixed point cell is constructed using ANSYS FLUENT. This is computational fluid dynamics (CFD) software which uses a finite volume method (FVM) to solve numerically a simplification of the equations that govern the physical problem in each volume in which the geometry is divided. This method was used in previous studies in this field [3]. The model describes the heat transport in a transient state during the freezing of the fixed point. Impurity segregation and grain growth were not modeled so only macroscopic thermodynamic effects were taken into account. 


\section{Influence of the Furnace Temperature Profile in the Freezing Plateau}

Three fixed points ( $\mathrm{Sn}, \mathrm{Zn}$ and $\mathrm{Al}$ ) were studied with different furnace conditions.

In the case of Sn, three very different furnace profiles with outer mantle initiation (see Figure 1) were performed. The objective was to compare the results with the ones obtained in previous studies [4] developed with a different model and software. It must be noticed that the furnace profiles in this study have been lineally extended to the cell full length because in the case of [4] only the part corresponding to the container of the fixed point element was simulated.

The furnace was initially set $1.3 \mathrm{~K}$ above Sn freezing point $(505.078 \mathrm{~K})$ and then cooled down to $1.3 \mathrm{~K}$ below the freezing point and from this offset $(503.778 \mathrm{~K})$ the furnace temperature profiles were added.

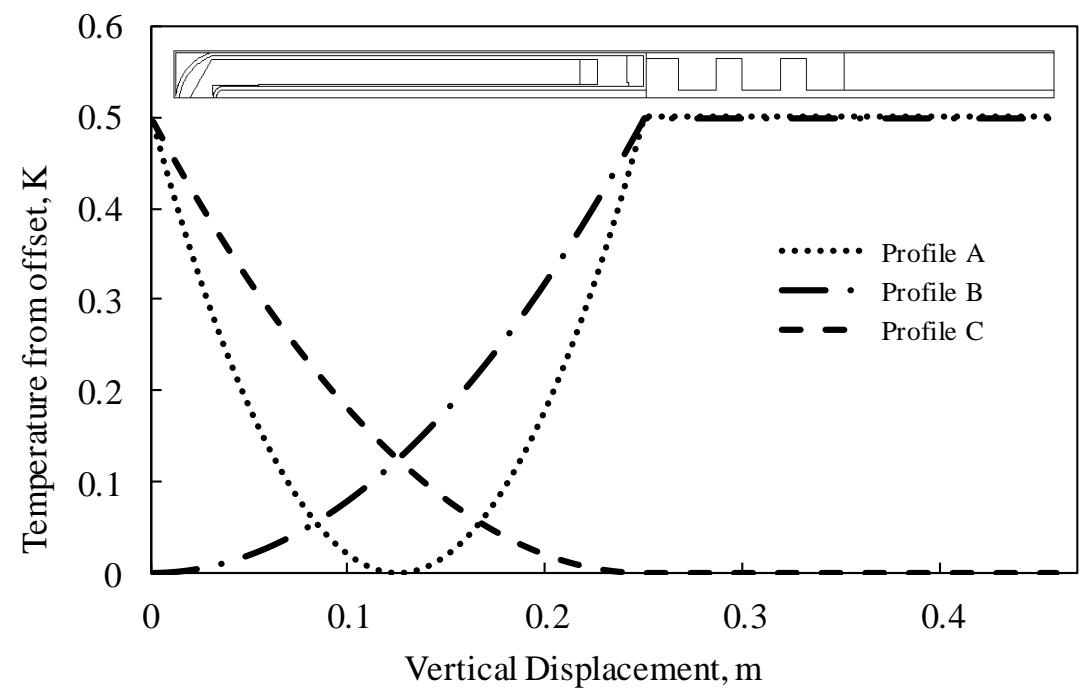

Fig 1. Furnace temperature profiles for Sn fixed point.

The freezing plateaus corresponding to these profiles are presented in Figure 2. These results are consistent with [1] i.e., a furnace that is warmer at the bottom of the cell minimizes the thermal effects.

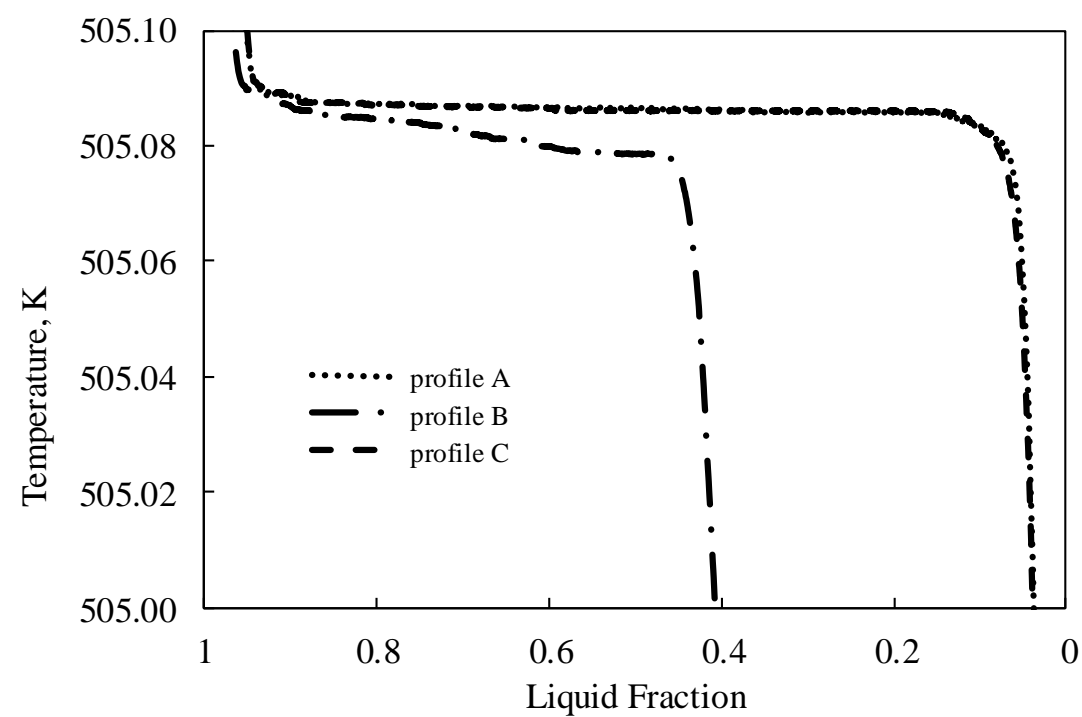

Fig 2. Freezing plateaus with the different furnace temperature profiles for Sn fixed point.

The influence of the furnace profile on the $\mathrm{Zn}$ point was studied comparing the fixed point realization in a furnace with only one heating resistance (single-zone furnace) with a thermal profile determined experimentally (see Figure 3), and the "ideal" case of a constant thermal profile of $1.5 \mathrm{~K}$ below the freezing point temperature. In both cases inner mantle initiation was considered. 


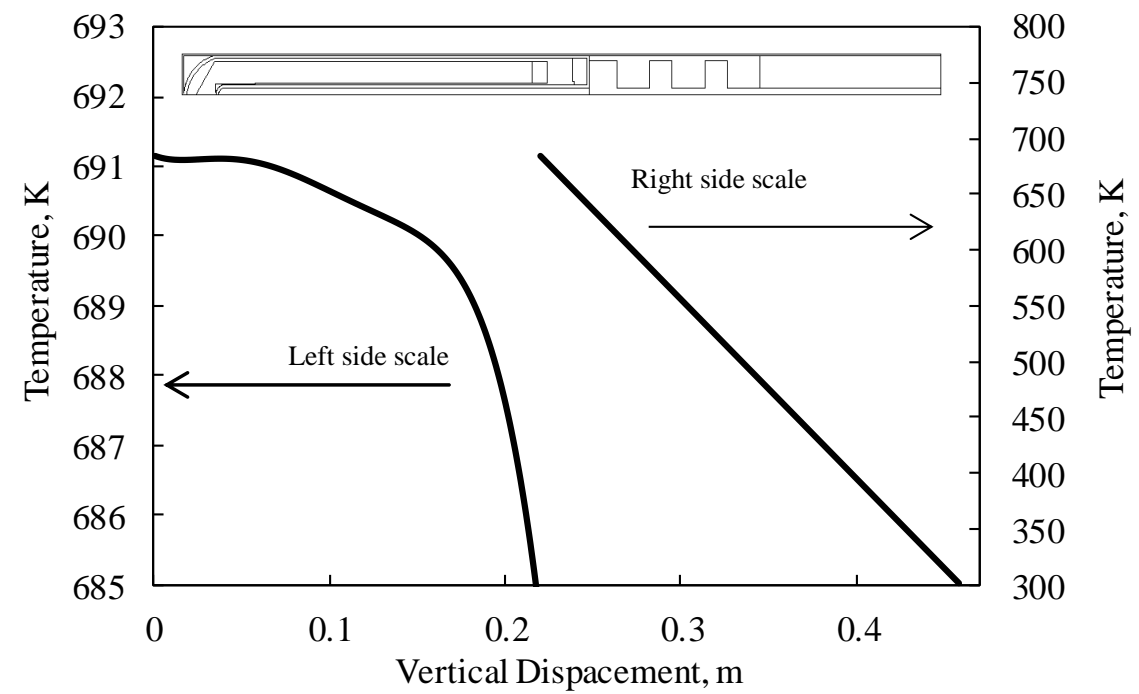

Fig 3. Single zone furnace temperature profile for $\mathrm{Zn}$ fixed point.

The inner mantle is initiated by two rods at room temperature introduced inside the thermometer well during one minute each. Freezing plateaus for the two conditions are plotted versus time in Figure 4. These results were compared with experimental realizations of a $\mathrm{Zn}$ cell in a single zone furnace and a three zone furnace that has and almost ideal profile. Figure 5 shows a relative plot of both plateaus. When comparing with figure 4 it can be seen than in both cases the plateau performed in a one zone furnace is about $25 \%$ to $30 \%$ shorter and realizes a fixed point temperature a bit lower than the temperature realized by the same cell in the furnace with the thermal profile closer to the ideal behavior (three zones).

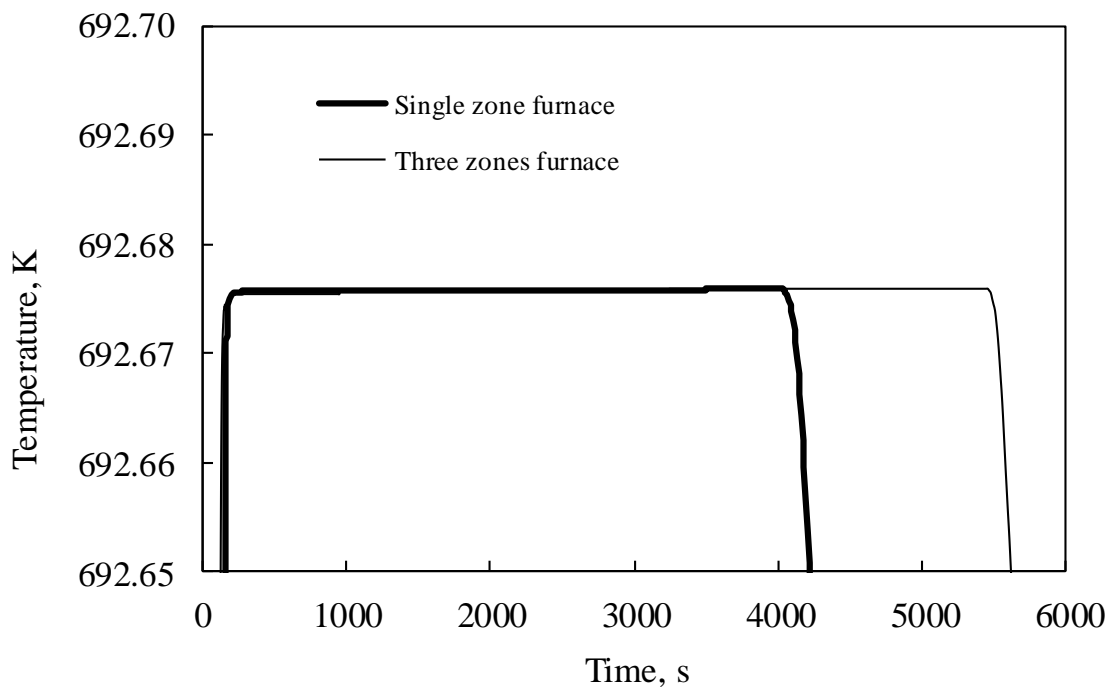

Fig 4. Zn freezing plateaus versus time modeled in a three zones and single zone furnaces. 


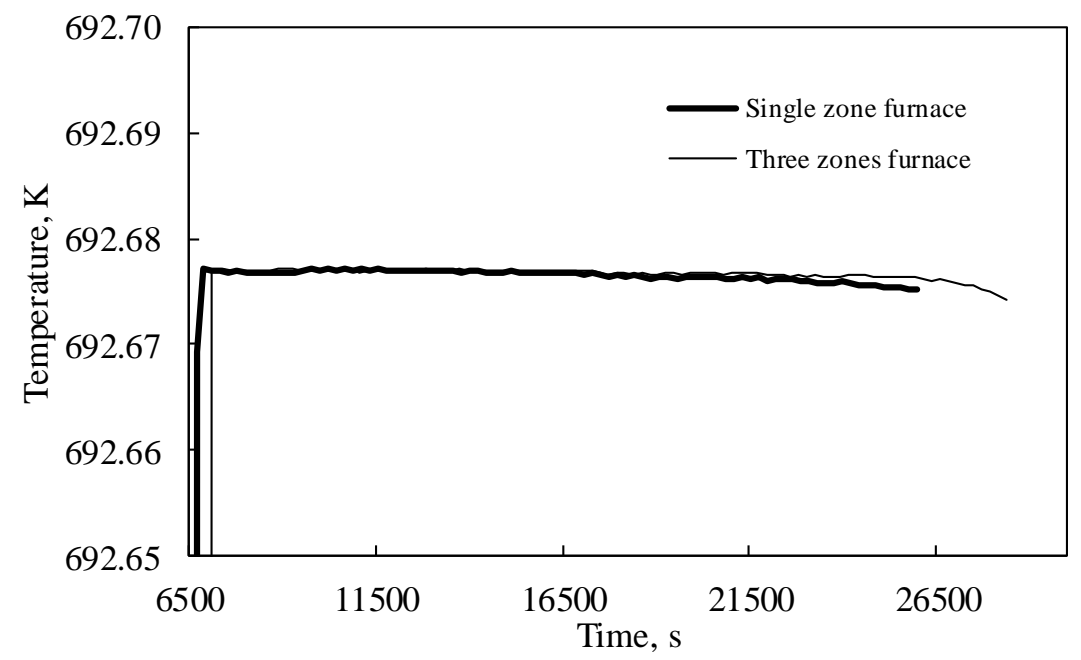

Fig 5. $\mathrm{Zn}$ freezing plateaus versus time measured in a three zones and single zone furnaces.

Finally, the influence of the furnace thermal profile in the realization of the $\mathrm{Al}$ fixed point was studied. Two different furnace designs were considered: three-zone and heat pipe, both having nearly equivalent (but different) profiles (Figure 6). Inner mantle initiation method was also used in this case.

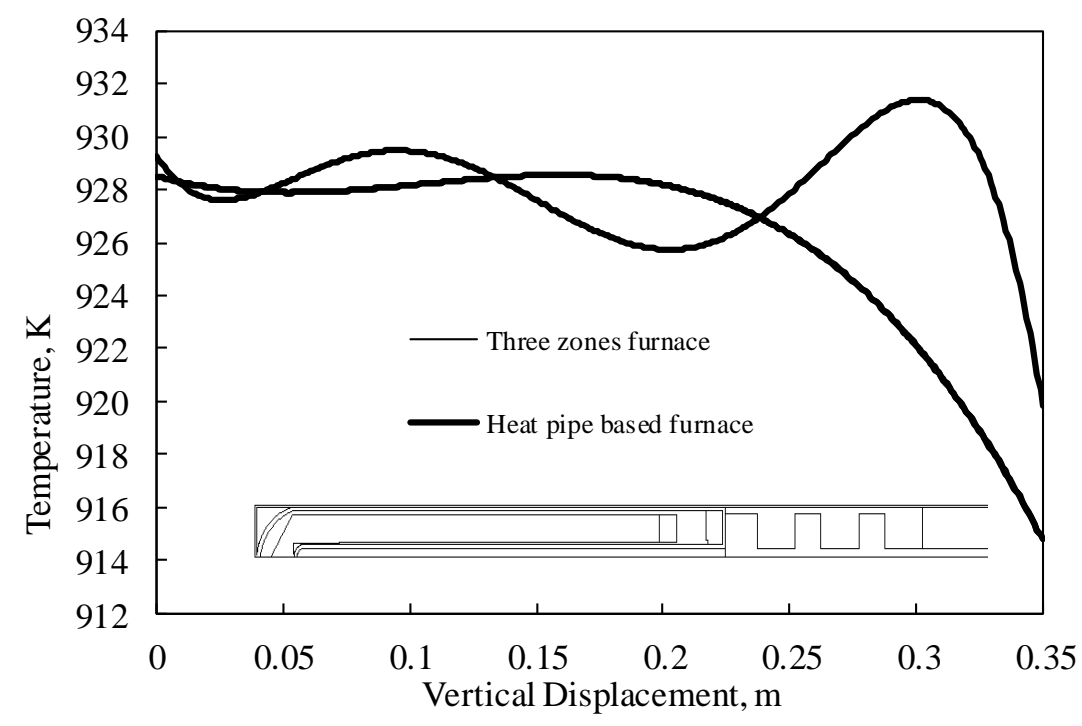

Fig 6. Furnace temperature profiles for Al fixed point.

For $\mathrm{Al}$ cell in these two furnaces, very little differences were found between both freezing plateaus as can be seen in Figure 7. 


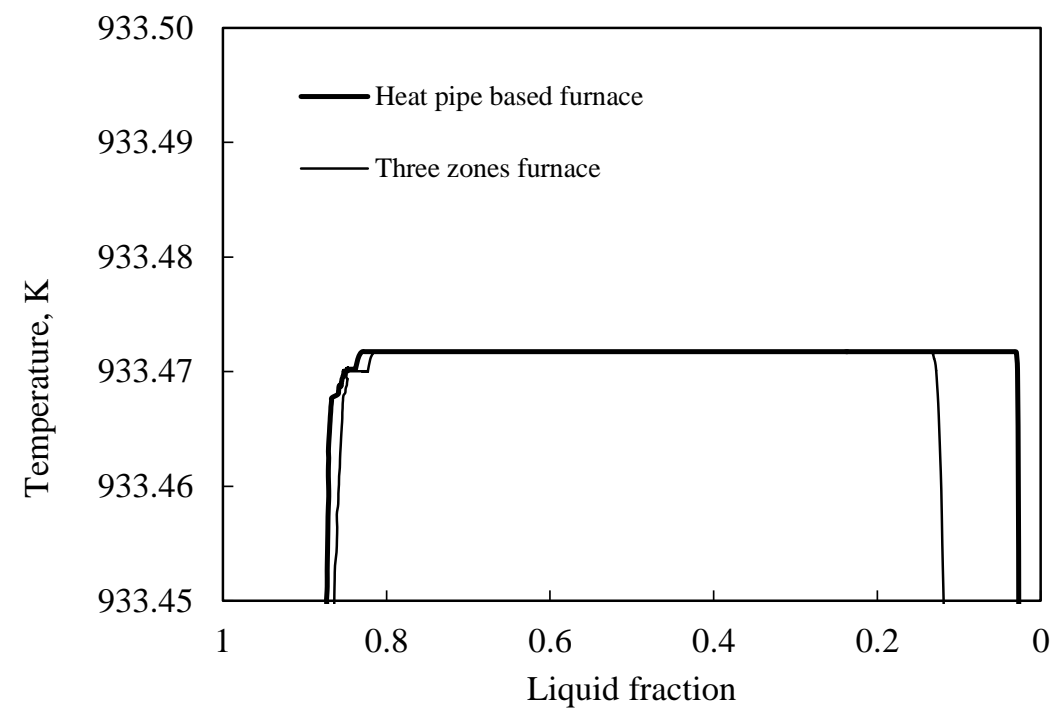

Fig 7. Freezing plateau versus liquid fraction with different furnace temperature profiles for $\mathrm{Al}$ fixed point.

\section{Influence of the Cell Design in the Freezing Plateau}

The results presented in the previous section were obtained for sealed fixed point cells that usually have external inconel containers. But a different design of fixed point cells is possible that uses quartz containers so as the cell is not sealed allowing the control and measurement of its inside pressure. In this case the correction of the measurements due to the internal pressure is possible allowing a more accurate realization of the fixed points because of the ITS-90 establishes a pressure of $101325 \mathrm{~Pa}$ in the case of the freezing points. Cells constructed with these two different designs have shown difference behaviors probably due to the different conductivity of inconel and quartz.

In this section, the influence of the external container material is observed in the case of the $\mathrm{Zn}$ point. Both cell designs were modelled inside a single and a three zone furnaces, giving four different study cases. The furnace profiles were the same as described in section 2. Figure 8 represents the freezing plateaus obtained by simulation of the behavior of these two different designs in a single zone furnace, while Figure 9 represents the experimental results obtained. Despite both results (simulation and experiment) are not fully comparable because the metal inside the two real cells comes from different batches, it can be seen that the behavior is similar, i.e. a longer freezing plateau for the open cell and a slightly high value for the closed cell. Figure 10 shows the simulation results obtained in a three-zone furnace. 


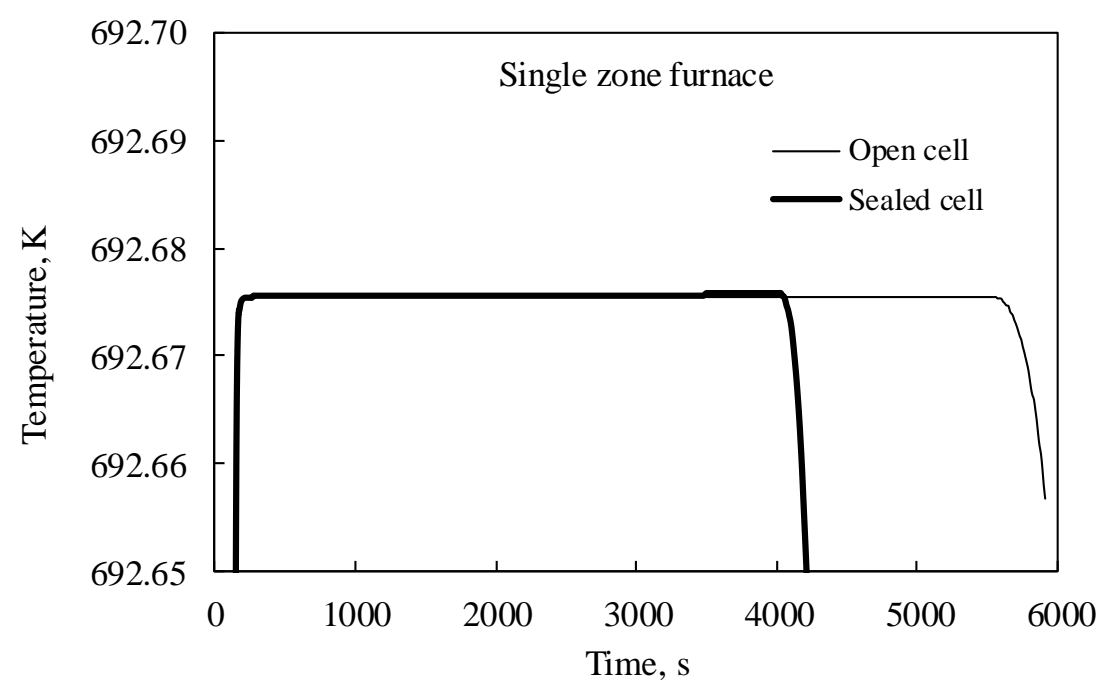

Fig. 8. Zn freezing plateau versus time modeled in a single zone furnace for sealed and open cells.

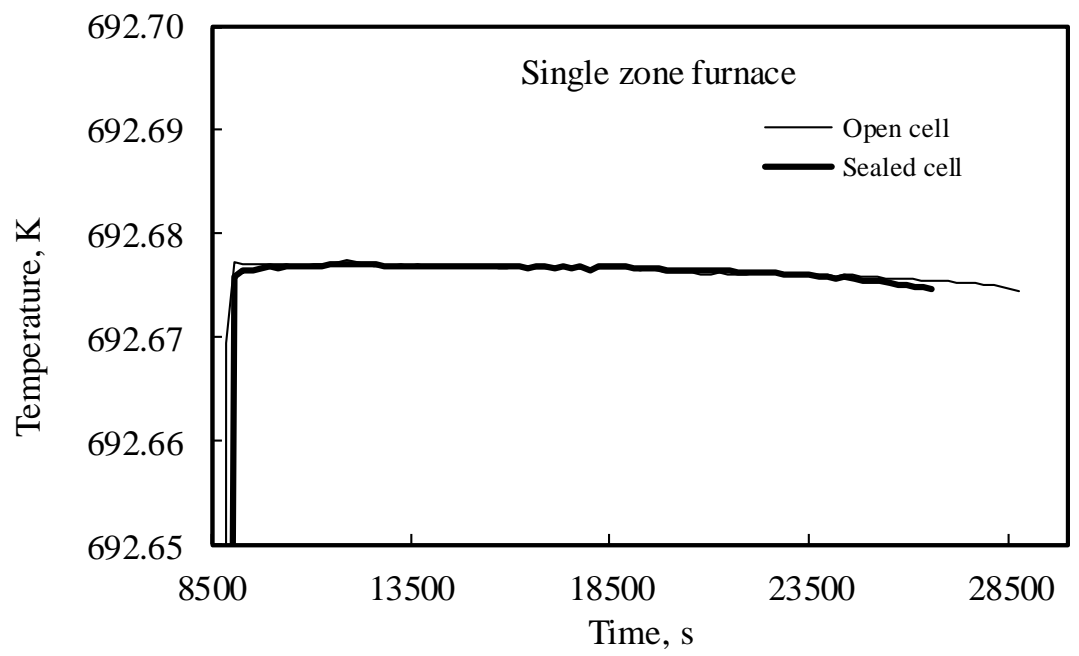

Fig 9. $\mathrm{Zn}$ freezing plateau versus time measured in a single zone furnace for sealed and open cells.

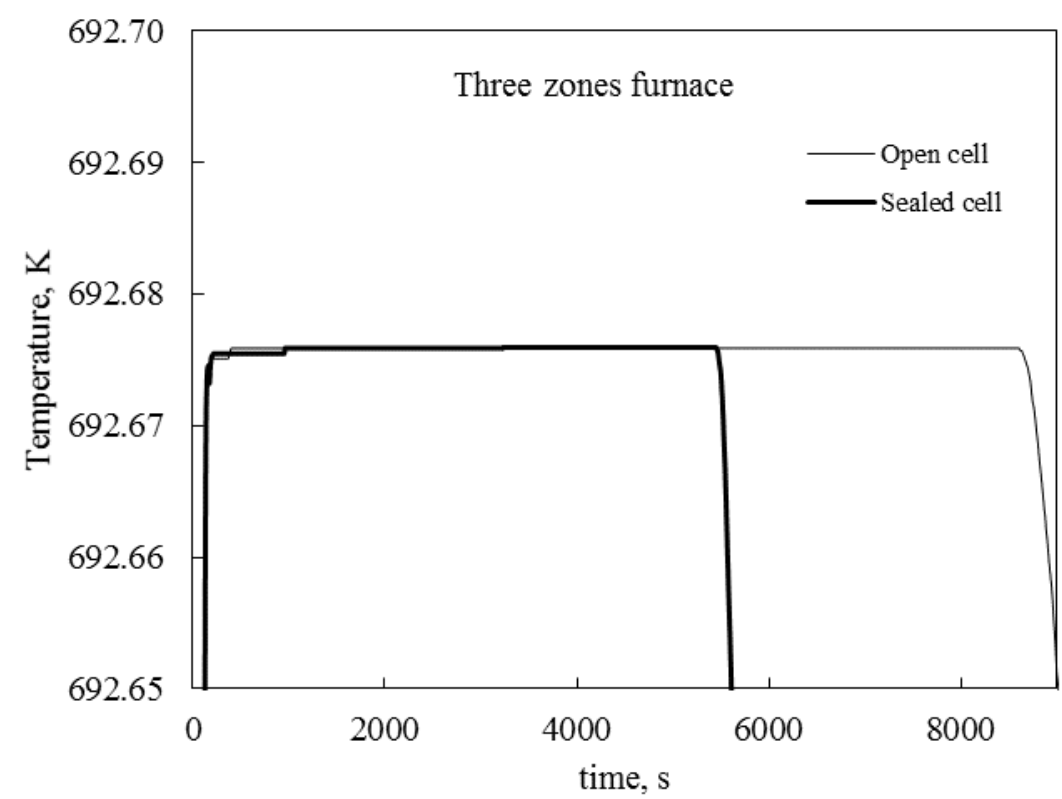

Fig. 10. Zn freezing plateau versus time modeled in a three zones furnace for sealed and open cells. 


\section{Influence of the Initiation Technique}

As was introduced in a previous section the realization of an outer and an inner liquid-solid interfaces in the fixed point cell would allow to obtain ideal thermal conditions. However differences in the realization procedure of these two mantles could lead to differences in the temperature of the fixed point realization. The reasons for this behavior are not clear and it is usually assumed that it is due to couplings between the furnace and the fixed point, impurity segregation effects, etc.

In [2] a new initiation technique for the metallic fixed points realizations is presented and compared with the traditional procedures. The technique consists of the realization of the inner mantle by the insertion of metallic rods (from one to three) cooled down to the liquid nitrogen temperature. It is not until the plateau is initiated that the furnace temperature is lowered to $0,4{ }^{\circ} \mathrm{C}$ below the freezing point temperature. The paper presents the comparison of the plateaus for the In and Sn fixed points obtained using this procedure and using the classical one, i.e. initiating the plateau by creating the outer interface lowering the furnace temperature about $1{ }^{\circ} \mathrm{C}$ and once the minimum of the supercooling is observed the inner interface is realize (by introducing cool rods at ambient temperature) and the furnace temperature set at $0,4^{\circ} \mathrm{C}$ below the freezing point temperature. In the case of In [2] shows that the plateaus obtained by the new method are longer and if, two cool rods used, the temperature realized by the cell is a bit higher than if only one rod is introduced in the thermometric well. This behavior is explained in [2] because of the microscopic behavior of the metals, i.e. crystal growth. These techniques have been simulated using the numerical model developed in this paper obtaining the plateaus plotted in Figure 11.



Fig. 11. Plateaus obtained for an In point realization using the method described in [2] using 1 and 2 metallic rods cooled at the liquid nitrogen temperature.

As expected the use of two rods instead of one makes shorter the freezing plateaus but unlike in the case of [2] the fixed point temperature realize when one rod is used is lower. This result highlights the inability of the model to deal with the fixed points microscopic effects like crystal growth. In order to be able to cope with these effects, the presence of impurities should be added using a Scheil diffusion model and a user-defined mathematical function incorporated to simulate the Gibbs-Thomson crystal growth effect.

\section{Conclusions}

Thermal fluxes are one of the major sources of uncertainty on the realization of the ITS-90 defining fixed points. The use of CFD can help to improve the understanding of the thermal exchanges that occurs between the cell and the furnace. 
In this paper a two-dimensional (2D) axisymmetric model of the furnace and the fixed point cell constructed using ANSYS FLUENT is presented. It has been validated using experimental results. The model has proven to be adequate to simulate macroscopic effects and allows to deep in the study of the interaction between the furnace and the cell while it is not to simulate microscopic effects like crystal growth.

As the paper shows the quantitative agreement between numerical and experimental procedures, the improvement is the ability to design optimal furnace conditions, cells and initiation techniques in a cheaper and faster way allowing to reduce the experimental procedures to the minimum required to realize the fixed points. .

\section{Acknowledgments}

This research is included in the EMRP Joint Research Project SIB10 NOTED, jointly funded by the EMRP participating countries within EURAMET and the European Union.

\section{References}

1. R. Morice, G. Bonnier, J.C. Barbaras, N. Fleurence, V. Le Sant, P. Ridoux, J.R. Filtz, Int. J. Thermophys. 29, 1785 (2008).

2. D. R. White, R. S. Mason, Int J Thermophys 32, 348 (2011).

3. P. Castro, G. Machin, M. A. Villamañan, D. Lowe, Int. J. Thermophys. 32, 1773 (2011).

4. M.J. Large, J. V. Pearce. Int. J. Thermophys. 35, 1109 (2014). DOI 10.1007/s10765-014-1685-2. 